\title{
Final Results from the High Resolution Fly's Eye (HiRes) Experiment
}

\author{
P. Sokolsky ${ }^{1}$ \\ Department of Physics and Astronomy \\ University of Utah \\ Salt Lake City, Utah \\ USA \\ E-mail:ps@physics.utah.edu
}

\section{For the HiRes Collaboration}

Final results from the High Resolution Fly's Eye (HiRes) on the observation of the Greisen-Zatsepin-Kuzmin cutoff in the cosmic ray spectrum are presented. We observe a cutoff consistent with the GZK predictions with a five sigma significance. The nature of the cosmic ray composition near the GZK cutoff is also discussed as well as possible correlations of the highest energy cosmic rays with AGN's and local large scale structure in the Northern sky.

35th International Conference of High Energy Physics (ICHEP2010)

Paris,France

July 22-28, 2

1 Speaker 


\section{Introduction}

Over the last forty years a variety of experiments have studied the cosmic ray spectrum at extreme energies[1]. This spectrum exhibits significant structure which must reflect the cosmic ray origins and propagation. Above energies of $10^{14} \mathrm{eV}$, the spectrum departs from a $\mathrm{E}^{-2.7}$ power law and steepens with a break at $10^{15} \mathrm{eV}$ known as the "knee." A second "knee" near $3 \mathrm{x}$ $10^{17} \mathrm{eV}$ has also been reported by a number of experiments. This is followed by an "ankle" structure with a dip near $3 \times 10^{18} \mathrm{eV}$. All this structure was predicted to culminate in a cutoff near $6 \times 10^{19} \mathrm{eV}$ beyond which the spectrum should drop abruptly. This final cutoff was predicted in 1966 by K. Greisen, G. Zatsepin and V. Kuzmin as the result of the inelastic interaction of protons with the 2.7 degree black body radiation[2]. However, the pioneering AGASA ground array in Japan presented evidence for a continuation of the spectrum beyond the GZK cutoff together with a lack of correlation with nearby astrophysical sources[3]. The High Resolution Fly's Eye (HiRes)[4] has produced data which now clearly shows the existence of a termination in the cosmic ray flux consistent with the GZK cutoff prediction. This measurement uses a pure air fluorescence technique. This observation has been recently confirmed by the Pierre Auger Observatory (PAO)[5] in Argentina which uses a combined ground array and air fluorescence detector

\subsection{The HiRes Experiment}

The HiRes experiment consists of two sites (HiRes I and II) $12.6 \mathrm{~km}$ apart, located at Dugway Proving Ground in the state of Utah. Each site consists of telescope units (22 at HiRes I and 42 at HiRes II) pointing at different parts of the sky. The detectors observe the full 360 degrees in azimuth but cover from 3 to 16.5 (Hires I) and from 3 to 30 degrees (HiRes II) in elevation angles. Since most cosmic ray events in this energy range are detected at distances of between 5 and $30 \mathrm{~km}$ from the detectors, higher elevation angles contribute little to the event rate. Each telescope consists of a $3.72 \mathrm{~m}^{2}$ effective area mirror and a 256 phototube camera cluster in the mirror focal plane. The phototubes subtend a one degree by one degree field of view on the sky. The tubes view signals through a UV filter which cuts out light below $300 \mathrm{~nm}$ and above $400 \mathrm{~nm}$ (corresponding to the air-fluorescence spectral range). The instantaneous aperture of the HiRes detector approaches $10,000 \mathrm{~km}^{2} \mathrm{str}$ at $10^{20} \mathrm{eV}$ but is limited by a 10 percent on time due to the requirement of dark, clear, moonless nights.

\subsection{Event Reconstruction}

The arrival direction of the cosmic ray initiating the shower can be reconstructed monocularly, using the triggered tube pointing directions to determine the shower-detector plane and relative tube trigger times, or by stereo, from the intersection of the two showerdetector planes from HiRes I and II. Details of the monocular and stereo reconstruction methods can be found in the literature[6][7]. Once the geometry of the event is determined, the tube signals are used to determine the shower size in one degree angular bins on the sky (for HiRes I), or in time bins corresponding to the FADC clock at HiRes II. Finally, combining the bin signal corrected for atmospheric attenuation with knowledge of the shower geometry, the size of the shower as a function of atmospheric depth is calculated. 


\section{Results on the spectrum, composition and anisotropy}

Fig. 1 shows the HiRes monocular[6]and stereo[7] spectra which clearly exhibit the "ankle" structure and a cutoff at the expected GZK energy. The figure also shows the result of fitting the monocular spectrum (which has the highest statistics) to three power laws with floating break points. The statistical strength of the monocular observation of the GZK cutoff is 5.3 sigma. The stereo spectrum, while more limited in statistics, has the best energy resolution due to the simplicity and robustness of the geometrical reconstruction. In addition, we have developed a series of cuts which make the stereo aperture essentially insensitive to variations in atmospheric transparency and other systematic errors.

Air fluorescence experiments determine composition by plotting the distribution of shower maxima, Xmax, as a function of energy and comparing the measured distribution with predictions for various assumptions about cosmic ray composition and hadronic interaction models[8]. The elongation rate and the absolute position of the mean Xmax and its fluctuations about the mean carry information about the primary composition. Model systematic uncertainty
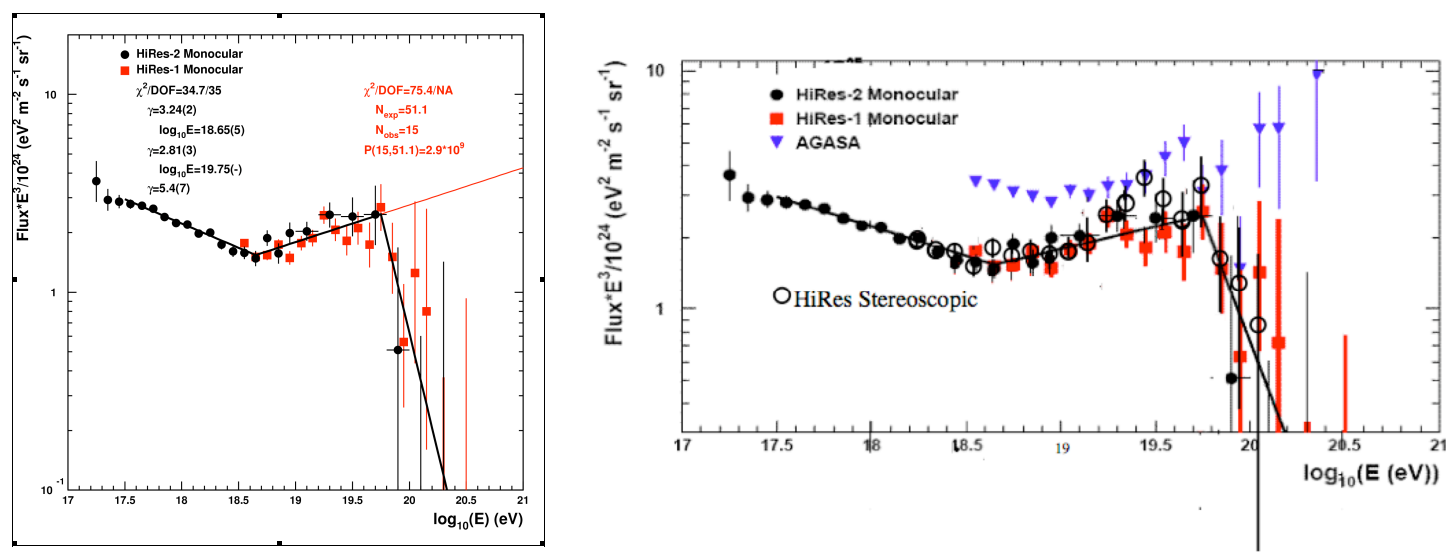

Figure 1: HiRes Monocular and Stereo Spectra. Triangles are data from the AGASA ground array which claimed a continuing spectrum beyond the predicted GZK cutoff. Figure on leftt shows power law fits to the spectrum and the statistical significance of the GZK cutoff determination.

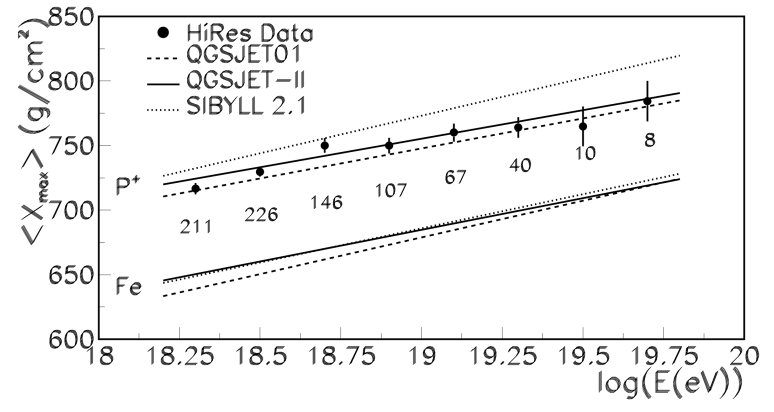

Figure 2: Elongation rate of showers compared to predictions from three hadronic interaction models for protons and for Fe nuclei. can be assessed by comparing the predictions of a variety of hadronic models and are discussed in reference 8 . The elongation rate is nearly model independent up to energies of 3 to $5 \times 10^{19}$ $\mathrm{eV}$ and the predictions of absolute mean $\mathrm{Xmax}$ position are in agreement to within $25-30 \mathrm{gm} / \mathrm{cm}^{2}$. Fig. 2 shows the elongation rate from the HiRes stereo experiment and model predictions[8]. Above $10^{18} \mathrm{eV}$, the data is consistent with a light, mainly protonic composition. 
Since the interaction of heavy nuclei will result in a superposition of showers, their fluctuations in Xmax should be significantly smaller than what is expected for protons. Fig 3 shows the HiRes fluctuation results in comparison with predictions of the QGSJET-II model. When comparing data to predictions, detector acceptance and trigger and reconstruction bias as well as resolution in energy and Xmax must be taken into acount. These effects are simulated by taking Corsika generated showers, putting them into a detailed detector simulation, creating simulated data in the same format as real data and reconstructing this simulated data using the same reconstruction programs used for real data. The calculated resolution in Xmax is shown in Fig. 4. This calculation can be checked by comparing the difference in shower maximum measured by HiRes I and HiRes II to the simulation predictions. HiRes I has a smaller vertical aperture and hence some events whose Xmax falls outside the aperture and are not well measured, generating a tail in the Xmax difference distribution. Nevertheless, the simulated events show exactly the same trend, confirming the accuracy of our simulation over the entire range of events (Fig. 4, left).

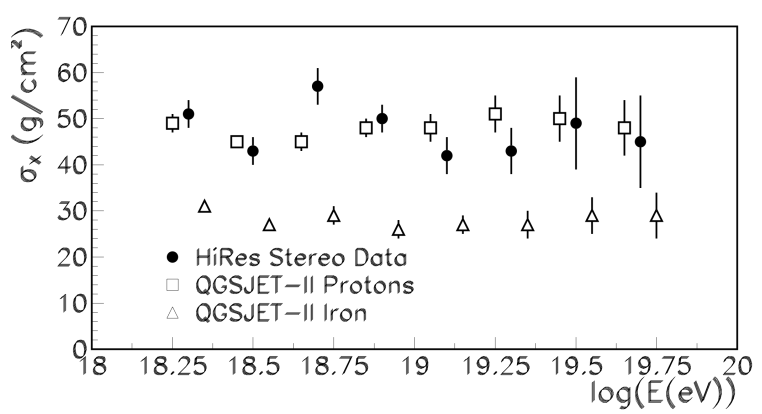

Figure 3: Fluctuations of showers about the mean Compared to predictions for protons and $\mathrm{Fe}$ for the QGSJET-II model. Predictions for other hadronic models are similar.
The HiRes data has been examined for correlation with nearby AGN's. The PAO experiment in the Southern Hemisphere published a possible correlation with AGNs in the Veron catalogue[9] with $\mathrm{Z}<.18$ and with cosmic ray energies above $10^{19.75}$. The correlation manifests itself as an excess of events within five degrees of the direction of the AGNs relative to what is expected for randoms.

The HiRes collaboration used the PAO parameters to search for an excess in the Northern hemisphere and has found nothing beyond what is expected from random coincidences with
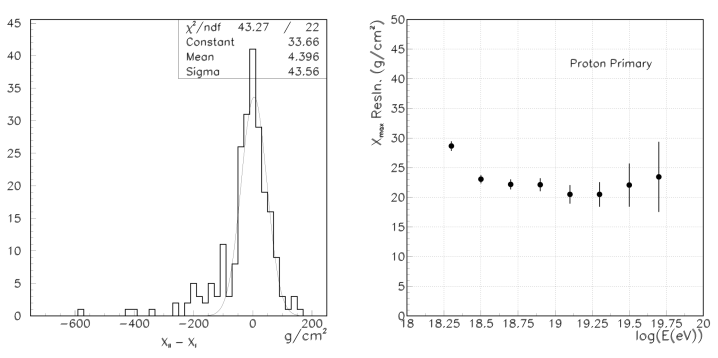

Figure 4: (Right) Xmax resolution as a function of energy. (Left) Xmax difference distribution between HiRes I and HiRes II for data. approximately the same number of events as the initial independent sample used by PAO[10]. Fig. 5 shows the distribution of HiRes stereo events in galactic coordinates and candidate AGN's from the Veron catalogue. Note that the HiRes acceptance is very different from the PAO acceptance. We have also looked for correlations with the local large scale structure (LSS) using a smoothed galaxy distribution from the $2 \mathrm{MRS}$ survey.

Fig. 6 shows event distributions relative to the LSS[11] No significant correlations with the LSS have been found at the $95 \%$ confidence level for events above $4 \times 10^{19} \mathrm{eV}$. Thus the sources of ultra-high energy cosmic rays in the Northern hemisphere remain mysterious. 

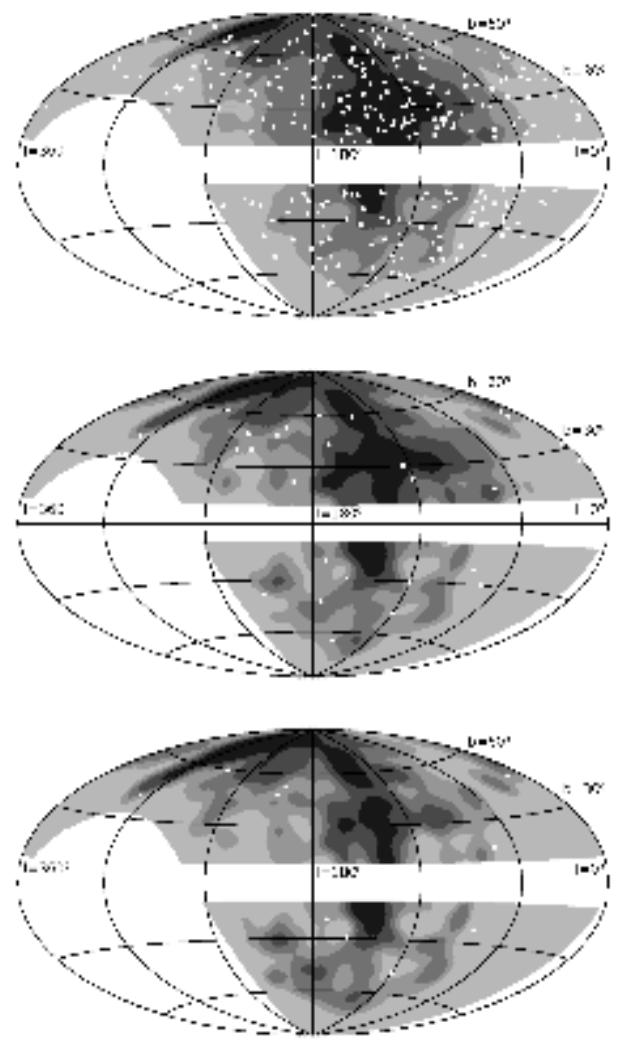

Figure 6: Search for correlation with smoothed LSS. Top, events with $>10^{19} \mathrm{eV}$, middle $4 \times 10^{19} \mathrm{eV}$, bottom $>6 \times 10^{19} \mathrm{eV}$. Events arrival directions are assumed to follow the LSS density out to $\mathrm{z}$ of 100 and are assumed isotropic beyond that.

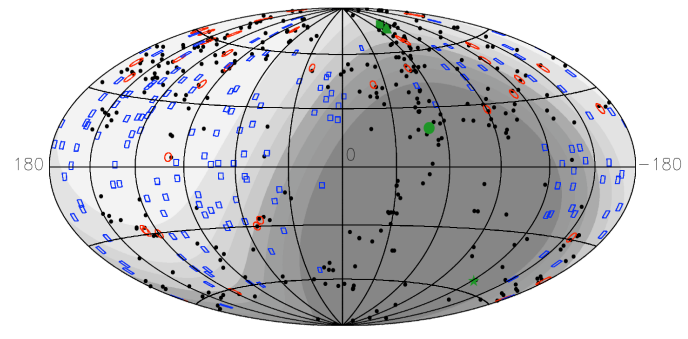

Figure 5: Distribution of arrival direction of stereo HiRes events versus nearby galaxies in the Veron catalogue. Black: AGN's, Blue: HiRes data, Red: Correlated data.

\section{Conclusion}

Significant progress has been made in the study of ultra-high energy cosmic rays. There is clear evidence for a termination of the spectrum at the energy predicted by Greisen, Zatsepin and Kuzmin more than 40 years ago. HiRes measurements of Xmax distributions are consistent with a light composition. The Pierre Auger Observatory confirms the cut-off but there are some differences interpreting the Xmax data. The new Telescope Array experiment[12] in Utah is in a good position to provide conclusive results.

\section{Acknowledgements}

This work is supported by US NSF grants PHY-9321949, PHY-9322298, PHY-9904048, PHY-9974537, PHY-0098826, PHY-0140688, PHY-0245428m OHT-0305516, PHY-0307098, and by the DOE grant FG03-92ER40732. We gratefully acknowledge the contributions from the technical staff of our home institutions and the cooperation of the US Army, and the Dugway Proving Grounds.

\section{References}

[1] P. Sokolsky and G. Thomson, J. Phys. G (2007).

[2] K. Greisen, Phys. Rev. Lett. 16, 48 (1968); T. Zatsepin and V. A Kuzmin, Pis'ma Zh. Eksp. Teor. Fiz.\}, 4, 114 (1966).

[3] M. Takeda et al., Phys. Rev. Lett. 81, 1163 (1998).

[4] G. Thomson et al., Nucl. Phys. B 36, 28 (2004).

[5] J. Abraham et al., Phys. Rev. Lett. 104, 091101 (2010).

[6] R. U. Abbasi et al., Phys. Rev. Lett. 92, 151101 (2004); R. U. Abbasi et al., Phys. Lett B 18, 271 (2005); R. U. Abbasi et al., Phys. Rev. Lett. 100, 101101 (2008).

[7] R. U. Abbasi et al., Astropart. Phys. 32, 5(2009).

[8] R. U. Abbasi et al., Phys. Rev. Lett. 104, 161101 (2010).

[9] J. Abraham et al., Science, 318, 938 (2008).

[10] R. U. Abbasi et al., Astropart. Phys. 30, 175 (2008).

[11] R. U. Abbasi et al., Ap. J. Letters 713 (2010) L64-L68.

[12] G. Thomson et al., in Proceedings of 35th Int. Conf. on High-Energy Physics, Paris, France, 2010. 